\title{
EVALUACIÓN DE FITOTOXICIDAD Y CARACTERIZACIÓN DE UN FERTILIZANTE LÍQUIDO ELABORADO MEDIANTE FERMENTACIÓN LÁCTICA UTILIZANDO SUBPRODUCTOS DEL PROCESAMIENTO DE TRUCHA (Oncorhynchus mykiss)
}

\section{PHYTOTOXICITY AND CHARACTERIZATION OF A LIQUID FERTILIZER PRODUCED BY LACTIC ACID FERMENTATION USING RAINBOW TROUT BY-PRODUCTS (Oncorhynchus mykiss)}

\author{
Marco Antonio Florez Jalixto ${ }^{1}$, David Julian Roldán Acero ${ }^{2}$ y \\ Juan Gabriel Juscamaita Morales ${ }^{3}$
}

\begin{abstract}
Resumen
El aumento de la producción de trucha en Perú genera gran cantidad de subproductos que generalmente no se reciclan, lo que causa gases de efecto invernadero, eutrofización cuando se arrojan al río, y aparición de plagas cuando se entierran. Los procesos de hidrólisis y fermentación láctica permiten recuperar aminoácidos y nutrientes, los cuales pueden ser empleados como suplemento para la fertilización foliar de plantas. El objetivo de la investigación fue elaborar un fertilizante líquido utilizando subproductos de trucha (FLVT), caracterizarlo y evaluar la fitotoxicidad. El contenido total de aminoácidos fue de $3.2 \mathrm{~g} / 100 \mathrm{~g}$ y el de proteína fue de $6.2 \mathrm{~g} / 100$ g; mientras el contenido de nitrógeno, fósforo y potasio fue de $12040 \mathrm{mg} / \mathrm{l}, 1189 \mathrm{mg} / \mathrm{l}, 5540 \mathrm{mg} / \mathrm{l}$, respectivamente. El fertilizante líquido no presentó E. coli ni Salmonella sp. Los contenidos de plomo, cadmio y cromo fueron menores a los límites máximos permisibles según las normativas para fertilizantes líquidos. En la prueba de fitotoxicidad en semillas de lechuga Lactuca sativa, las concentraciones del FLVT de $0.1 \%$ a $0.001 \%$ estuvieron libres de sustancias fitotóxicas y con valores de índice de germinación (IG) mayores a $80 \%$.
\end{abstract}

Palabras clave: fertilizante líquido, fitotoxicidad, fermentación láctica, vísceras de trucha, perfil de aminoácidos.

\begin{abstract}
The increase of rainbow trout production in Peru generates a large amount of by-products which are not generally recycled, which generates greenhouse gases, eutrophication when they are thrown into the river and the appearance of pests when they are buried. The processes of hydrolysis and lactic acid fermentation allow to recover amino acids and nutrients, which can be used as a supplement for foliar fertilization of plants. The aim of this research was to develop a liquid fertilizer using rainbow trout by-products (FLVT), characterize it and evaluate the phytotoxicity. The total amino acid content was $3.2 \mathrm{~g} / 100 \mathrm{~g}$ and protein content was $6.2 \mathrm{~g} / 100 \mathrm{~g}$. While the content of nitrogen, phosphorus and potassium were $12040 \mathrm{mg} / \mathrm{l}, 1189 \mathrm{mg} / \mathrm{l}, 5540 \mathrm{mg} / \mathrm{l}$, respectively. The liquid fertilizer did not contain $E$. coli or Salmonella sp. While the content of heavy metals such as lead, cadmium and chromium were lower than the maximum permissible limits according to the regulations for liquid fertilizers. In the phytotoxicity test on Lactuca sativa lettuce seeds, the concentrations of FLVT from $0.1 \%$ to $0.001 \%$ were free of phytotoxic substances and germination index values (IG) were greater than $80 \%$.
\end{abstract}

Key words: liquid fertilizer, phytotoxicity, lactic acid fermentation, rainbow trout viscera, amino acid profile.

\section{Introducción}

El incremento de la producción pesquera mundial en los últimos años ha causado el aumento en la generación de residuos. En el Perú, según PRODUCE (2018), la trucha arco iris representó el $63.79 \%$ del total de recursos hidrobiológicos de origen continental y su extracción, proveniente de la acuicultura, fue de 57 210.69 t en 2017, siendo destinadas 53734.27 t como fresco y 3475.80 t para congelado. Actualmente, los mercados nacionales exigen un alto grado de frescura, debido a ello, los productores ofertan la trucha como fresco eviscerado. Durante el procesamiento de trucha se generan distintos subproductos y desechos que corresponden al $19 \%$ del peso vivo (Torres et al., 2013). El eviscerado implica la remoción de los órganos internos, así como también la limpieza de la 
cavidad corporal del peritoneo, tejido renal y sangre. De tal forma que las vísceras constituyen de 5 a $8 \%$ del peso del pescado entero (Waterman, 2001). Por otro lado, el reciclaje de residuos orgánicos es importante para la recuperación de nutrientes y compuestos orgánicos de la biomasa no utilizada de los diferentes recursos, evitando la generación de gases de efecto invernadero cuando estos residuos se entierran, arrojan a ríos o se colocan en rellenos sanitarios (Hwang et al., 2015).

Los subproductos son fuente de proteína, calcio, vitaminas, hierro y minerales (Aranganathan \& Rajasree, 2016) y debido a su contenido de aminoácidos pueden ser utilizados como harina de pescado, salsa de pescado, fertilizantes o ensilado (Hamid et al., 2002). La forma convencional de reutilizar subproductos de pescado es la transformación en harina de pescado, sin embargo, este proceso es costoso, además que sobrecalienta la materia prima y el proceso de secado disminuye la digestibilidad de la harina (Yamamoto et al., 2005). La harina de restos de pescado es utilizada en alimentación animal, su forma de elaboración dependiendo de la tecnología utilizada puede ocasionar quemado, oxidación y pérdida de nutrientes, entre ellos proteínas, aminoácidos, vitaminas y minerales. Por otro lado, el compostaje es un bioproceso lento llevado a cabo por microorganismos, tiene una duración que puede variar desde algunas semanas hasta varios meses e incluye cuatro fases: una fase de descomposición inicial, una fase termofílica de intensa descomposición microbiana, una segunda fase termofílica y una fase de maduración (Goldhor \& Regenstein, 2007; Radziemska et al., 2019), debido a ello, requiere de mayor número de operarios para realizar actividades como el montaje de pilas de compost en capas, homogenización y cambio de capas del sustrato, adición de agua, control de humedad y de aireación. Adicionalmente, se controlan parámetros como: temperatura de la biomasa, humedad, pH, cenizas, índice de mineralización, materia orgánica, carbono total, nitrógeno total y relación carbono:nitrógeno que debe ser de 25:1 a 30:1. Una pila de compost de pescado debe tener una humedad de $40 \%$ a $60 \%$, ya que un exceso de agua limita la difusión del oxígeno; mientras que la temperatura de la biomasa, que disminuye progresivamente, alcanza los $23{ }^{\circ} \mathrm{C}$ luego de 60 días (Valente et al., 2017; Lopes et al., 2019). En cambio, la fermentación láctica es un bioproceso más rápido y práctico que sólo necesita homogenización diaria y controlar el descenso de $\mathrm{pH}$ hasta 4 , lo cual se logra en 5 días a $40{ }^{\circ} \mathrm{C}$ (Cupe \& Juscamaita, 2018).

Los fertilizantes orgánicos líquidos están conformados por una variedad de materiales que provienen de fuentes de origen vegetal y animal, incluyen hidrolizados de pescado, emulsiones de pescado y de macroalgas. La utilización del hidrolizado de proteína de pescado como fertilizante líquido (FL) se ha estudiado con la finalidad de aumentar el valor nutritivo, compuestos bioactivos y actividad antioxidante de muchas plantas (Fernández et al., 2015).

Aranganathan \& Rajasree (2016) mencionan que la utilización de residuos y subproductos de pescado en la producción de fertilizantes orgánicos para fines agrícolas es muy limitada. Sanes et al. (2015) señala que el aprovechamiento de los subproductos de pescado es una fuente de nutrientes que puede utilizarse en la elaboración de FL para ser empleados en la producción agrícola sostenible por empresas familiares. Además de ser una alternativa para conseguir ingresos económicos mediante su comercialización por los agricultores, o bien puede ser usado en sus propios cultivos (Unnisa, 2015). La fermentación microbiana es un proceso fácilmente adaptable a menor y mayor escala que incluye procedimientos relativamente simples (Owens \& Mendoza, 1985). Debido a esto, ha sido propuesta para el procesamiento de subproductos y desechos de pescado. Además, de que un sistema anaerobio no requiere de mucha mano de obra en comparación con un sistema aerobio (Sanes et al. 2015).

Valente et al. (2016) utilizó residuos del fileteado de pescados marinos para elaborar compostaje, el cual es una tecnología eficiente para la descomposición de residuos generados por la industria pesquera, y fue utilizado como fertilizante orgánico. Mientras que Casaclang et al. (2017) elaboraron un FL, mediante fermentación biológica, a partir de subproductos de tilapia y sabalote. Así mismo, Sanes et al. (2015) realizaron un proceso de compostaje y fermentación de residuos de pescado.

El objetivo de la investigación fue elaborar un fertilizante orgánico líquido utilizando subproductos de la trucha (siendo las vísceras el principal subproducto del procesamiento en granjas piscícolas), caracterizarlo con la finalidad de reportar su contenido de aminoácidos, macronutrientes y micronutrientes que permitirían conocer el potencial de su aplicación como suplemento en la fertilización de plantas y evaluar el índice de fitotoxicidad del FLVT.

\section{Materiales y Métodos}

En la elaboración del FL se utilizaron los subproductos (estómago, intestino, hígado, corazón, sangre y gónadas) derivados del eviscerado de la trucha, en la región Canta, Lima. El flujo de proceso fue el siguiente: recepción de vísceras de trucha molido - mezclado - hidrólisis enzimática con proteasa a $0.33 \%(\mathrm{p} / \mathrm{v})$ y a $60{ }^{\circ} \mathrm{C}$ - inactivado a $85{ }^{\circ} \mathrm{C}$ por 20 minutos - colado - mezclado de hidrolizado de vísceras con melaza y con un consorcio microbiano comercial Bio-Lac (cultivo de bacterias lácticas) como inóculo incubado a $40{ }^{\circ} \mathrm{C}$ por 5 días - colado - envasado. 
Tratamientos y análisis de datos

Se empleó un diseño completamente al azar (DCA) con arreglo factorial el cual se basó en la combinación de 3 niveles $(5 \%, 10 \%$ y 15\%) de inóculo (Factor 1) y 4 niveles $(5 \%, 10 \%, 15 \%$ y $20 \%$ ) de melaza (Factor 2). La variable respuesta fue el $\mathrm{pH}$ luego de transcurridos 5 días de fermentación láctica a $40{ }^{\circ} \mathrm{C}$. La Normalidad de los errores y homogeneidad de varianza fueron comprobadas mediante las pruebas de Shapiro Wilks modificadas y de Levene, respectivamente. Se realizó un análisis de varianza (ANVA) y una prueba comparativa LSD Fisher $(\mathrm{p}<0.05)$ utilizando el software estadístico R.

Métodos analíticos

El perfil de aminoácidos se obtuvo empleando un cromatógrafo de gases (HP 6890, HEWLETT PACKARD, USA). El contenido de aminoácidos ( $\mathrm{g}$ de aminoácido/100 g muestra) se realizó mediante la metodología descrita por Heinrikson \& Meredith (1984) y el contenido de triptófano (g triptófano/100 g muestra) mediante la metodología reportada por Hugli $\&$ Moore (1972).

$\mathrm{El} \mathrm{pH}$ y la conductividad eléctrica se determinaron utilizando el Potenciómetro C860 (Consort, Bélgica) y el Conductímetro 32 (Ysi, USA), respectivamente. El índice de acidez (\%) se determinó por el método propuesto por Berenz (1997).

La determinación de nitrógeno total $(\mathrm{mg} / \mathrm{l})$ se realizó utilizando un digestor Turbotherm (Gerhardt, Alemania). La determinación de fosforo total (P) se realizó mediante una digestión vía húmeda y posterior colorimetría empleando el método sulfomolíbdico utilizando un espectrofotómetro UV Genesys 20 (Thermo Scientific, USA). La determinación total $(\mathrm{mg} / \mathrm{l})$ de potasio $(\mathrm{K})$, calcio $(\mathrm{Ca})$, magnesio $(\mathrm{Mg})$, sodio $(\mathrm{Na})$, hierro $(\mathrm{Fe})$, cobre $(\mathrm{Cu})$, zinc $(\mathrm{Zn})$, manganeso $(\mathrm{Mn})$, cromo $(\mathrm{Cr})$, cadmio $(\mathrm{Cd})$ y plomo $(\mathrm{Pb})$ se realizó utilizando un espectrofotómetro de absorción atómica (Aanalyst 200 AA, Perkin, USA). Mientras que la determinación de boro (B) se realizó mediante calcinación utilizando una mufla 48000 (Thermolyne, USA). Todos los análisis se realizaron según las metodologías de Chapman \& Pratt (1973).

Los ensayos microbiológicos se realizaron siguiendo las recomendaciones de ICMSF (ICMSF, 1983). En el recuento de Lactobacillus sp. (UFC/ml) se utilizó el medio Agar MRS incubado en condiciones anaeróbicas a $30{ }^{\circ} \mathrm{C}$ por 5 días. El recuento de mohos y levaduras (UFC/ml) fue en medio Agar OGY, incubado a 20 - $24{ }^{\circ} \mathrm{C}$ por 3 a 7 días. La enumeración de Escherichia coli $(\mathrm{NMP} / \mathrm{ml})$ se realizó en medio Caldo Brilla Fluorocult incubado a $45{ }^{\circ} \mathrm{C}$ por 48 horas, y la detección de Salmonella sp fue en $25 \mathrm{~g}$ de muestra.

La prueba de fitotoxicidad se realizó utilizando semillas de lechuga (Lactuca sativa) variedad Duett, siguiendo la metodología propuesta por Sobrero \& Ronco (2008). Para el cálculo del índice de germinación (IG, Fórmula 1) se siguió la metodología seguida por Zucconi et al. (1981). Se calculó el porcentaje de germinación relativo (PGR, Fórmula 2) y el crecimiento de radícula relativo (CRR, Fórmula 3) para cada dilución y se usó agua destilada control. Las ecuaciones utilizadas fueron:

$$
\mathrm{IG}=\frac{\mathrm{PGRxCRR}}{100}
$$

Fórmula 1. Índice de Germinación (IG)

$$
\text { PGR }=\frac{\mathrm{N}^{\circ} \text { de semillas germinadas en la dilucion }}{\mathrm{N}^{\circ} \text { de semillas germinadas en el control }} \times 100
$$

Fórmula 2. Porcentaje de Germinación Relativo (PGR).

$$
\mathrm{CRR}=\frac{\text { Elongacion de radícula en la dilución }}{\text { Elongacion de radícula en el control }} \times 100
$$

Fórmula 3. Crecimiento de Radícula Relativo (CRR).

El IG es la variable que integra diferentes grados de fitotoxicidad y representa un indicador robusto para describir el potencial fitotóxico de un material orgánico (Varnero et al., 2007).

\section{Resultados y discusión}

El sustrato, hidrolizado enzimático de vísceras de trucha, mezclado con melaza e inóculo registró valores de $\mathrm{pH}$ inicial de 6 a 7, luego del quinto día, el pH de los tratamientos registró valores de 3.4 a 3.91, como se observa en la Tabla 1. La fuente de $\mathrm{N}$ en forma de proteínas permitió la hidrólisis microbiana del sustrato. La melaza como fuente de carbono y energía, permitió el crecimiento y desarrollo de bacterias lácticas, lo cual tuvo como consecuencia el descenso de $\mathrm{pH}$ hasta el punto de que el ácido láctico producido inhibió el crecimiento de las propias bacterias lácticas (Soni et al., 2013).

El ANVA de la fermentación láctica del sustrato, luego de transcurridos cinco días, señaló que no existe interacción entre los factores: melaza e inóculo. La prueba comparativa de medias (LSD Fisher) de las proporciones $5 \%, 10 \%$ y $15 \%$ de melaza presentaron diferencias significativas, sin embargo, no hubo diferencias significativas entre las proporciones de $15 \%$ y $20 \%$, de igual manera tampoco hubo diferencias significativas entre las proporciones de $10 \%$ y $20 \%$. Al respecto, Sahu et al. (2017) señalan que una proporción de 7 a $10 \%$ de melaza o dextrosa garantiza una adecuada producción de ácido láctico por parte de las bacterias lácticas. Respecto a la proporción de inóculo, no hubo diferencias significativas entre las proporciones de $10 \%$ y $15 \%$. En la Figura 1 se muestra la variación de $\mathrm{pH}$ y acidez titulable de los tratamientos $\mathrm{T}_{5}, \mathrm{~T}_{9}$ у $\mathrm{T}_{12}, \quad$ los cuales presentaron mejores características sensoriales durante la estabilización de las vísceras de trucha hidrolizadas. Con la finalidad de reutilizar la mayor cantidad de subproducto y minimizar la cantidad de inóculo, se fijaron 

Agosto - Diciembre 2020

proporciones para el mezclado con el sustrato de $10 \%$ $(\mathrm{p} / \mathrm{p})$ melaza y $10 \%(\mathrm{p} / \mathrm{p})$ inóculo.

Tabla 1. Evaluación del pH luego del quinto día de fermentación láctica de vísceras hidrolizadas de trucha.

\begin{tabular}{|c|c|c|c|}
\hline 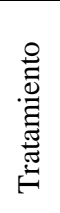 & 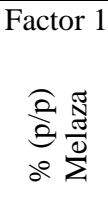 & 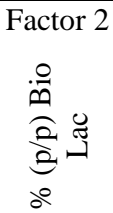 & 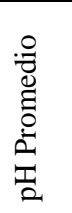 \\
\hline $\mathrm{T}_{1}$ & 5 & 5 & 3.91 \\
\hline $\mathrm{T}_{2}$ & 10 & 5 & 3.76 \\
\hline $\mathrm{T}_{3}$ & 15 & 5 & 3.6 \\
\hline $\mathrm{T}_{4}$ & 5 & 10 & 3.87 \\
\hline $\mathrm{T}_{5}$ & 10 & 10 & 3.66 \\
\hline $\mathrm{T}_{6}$ & 15 & 10 & 3.55 \\
\hline $\mathrm{T}_{7}$ & 5 & 15 & 3.69 \\
\hline $\mathrm{T}_{8}$ & 10 & 15 & 3.59 \\
\hline $\mathrm{T}_{9}$ & 15 & 15 & 3.4 \\
\hline $\mathrm{T}_{10}$ & 20 & 5 & 3.64 \\
\hline $\mathrm{T}_{11}$ & 20 & 10 & 3.64 \\
\hline $\mathrm{T}_{12}$ & 20 & 15 & 3.62 \\
\hline
\end{tabular}

La fermentación láctica logró biotransformar los subproductos (vísceras principalmente) generados en el procesamiento primario de eviscerado de trucha (entre $10 \%$ a $15 \%$ del peso de la trucha entera), que es efectuado en la sierra del país. Según PRODUCE (2018), las mayores extracciones de trucha destinada para consumo en estado fresco fueron realizadas en Puno, con 45048.01 t, Junín con 2610.36 t, Huancavelica con 1980.28 t, Cusco 1287.22 t. Por lo tanto, el método biológico propuesto podría cubrir la necesidad de darle valor agregado a los subproductos de la principal especie acuícola en dichas regiones. Además, en los departamentos antes mencionados, se realiza una importante actividad agrícola, que permitiría su utilización.

Los métodos para biotransformar biomasa de residuos pesqueros en FL incluyen desde bioprocesos complejos, como biodegradación aeróbica, hasta métodos más prácticos como fermentación láctica y endógena. Por ejemplo, Kim et al. (2010) y Figueroa et al. (2015) emplearon como consorcio microbiano bacterias aisladas de vísceras de lombrices de tierra, compost y humus comercial; además, utilizaron como proceso una biodegradación aeróbica (llevado a cabo en biorreactor), que implica controles de parámetros de la reacción: $\mathrm{pH}$, flujo de oxígeno disuelto y potencial de oxidación-reducción. Por otro lado, Hepsibha \& Geetha (2019) y Ramesh et al. (2020) emplearon una fermentación heteroláctica, llevada a cabo por las enzimas endógenas de los residuos de pescado y solo con la adición de panela como fuente de carbono; como resultado, hubo una producción de ácido láctico, ácido acético, etanol y $\mathrm{CO}_{2}$, lo cual puede inducir a una formación de gas que puede ocasionar inconvenientes en su almacenamiento luego de ser envasado. El método utilizado consistió en una fermentación homoláctica debido al consorcio microbiano comercial (Bio-Lac) compuesto por bacterias lácticas de los géneros Lactobacillus, Streptococcus y Bifidobacterium.

Evaluación organoléptica

El FLVT elaborado presentó un olor ligero a aceite de pescado y melaza, un color ámbar oscuro y una

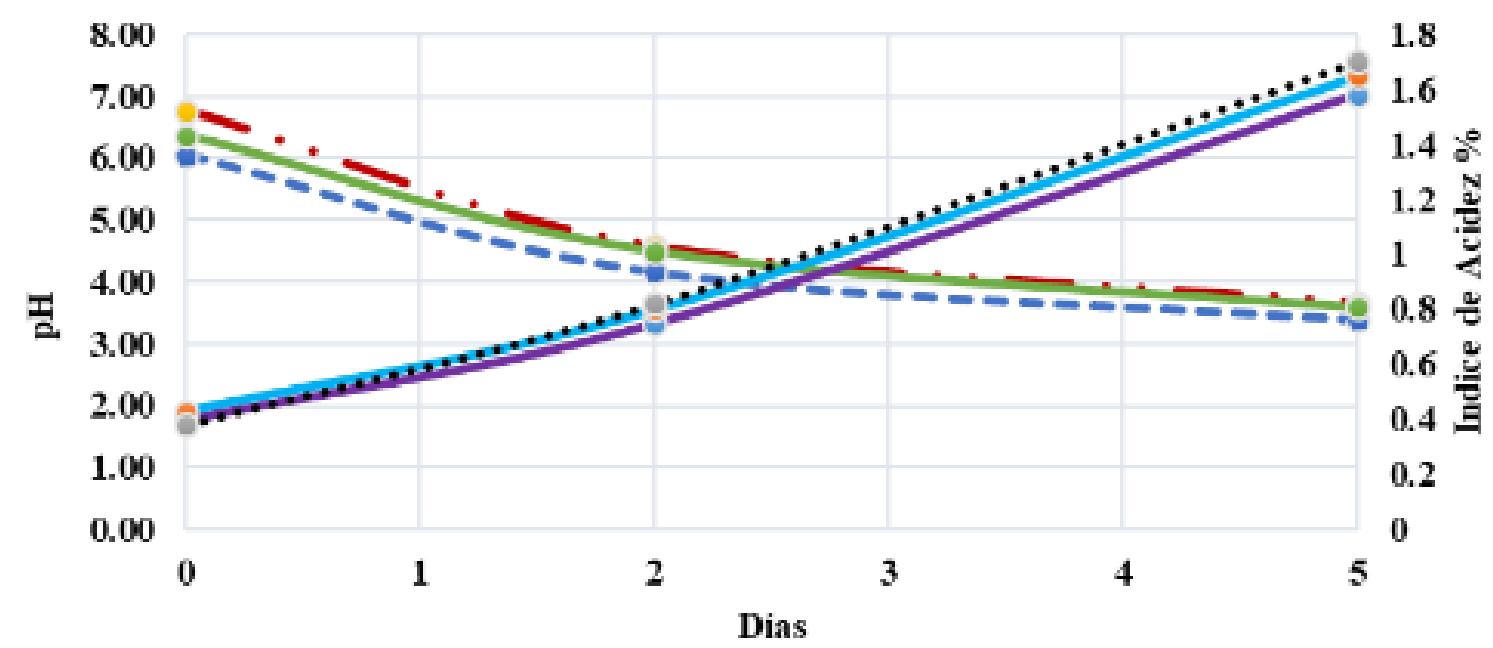

— pHT5 $\because-$ pHT9 $-\bullet-$ pHT12 $-\bullet$ T5 $-\bullet$ T9 $\cdots \bullet$ T12

Figura 1. Variación de $\mathrm{pH}$ y acidez de los tratamientos $\mathrm{T}_{5}, \mathrm{~T}_{9}$ y $\mathrm{T}_{12}$ durante los 5 días que ocurrió la fermentación láctica. 
consistencia líquida, según los parámetros del análisis sensorial para ensilado biológico de pescado (Holguín et al., 2009). La adición de $20 \%$ de melaza fue suficiente para obtener un FL con buen olor y color marrón oscuro (Sastro et al., 2013). Aranganathan \& Rajasree (2016) elaboraron un FL con olor agradable, agregando $10 \%$ de melaza y Bacillus subtilis en la fermentación de residuos de pescado de la familia Cupleidae.

Análisis físico químico

Los resultados de la composición físicoquímica del FLVT son mostrados en la Tabla 2. Ito (2006) señala que el $\mathrm{pH}$ cercano a 4.2 permite la estabilización del proceso de fermentación en la elaboración de fertilizantes orgánicos. De hecho, la fermentación de subproductos del fileteado de tilapia, con $3 \%$ (v/p) Lactobacillus casei y $15 \%$ de melaza, logra estabilidad a un pH cercano a 4.5 (Spanopoulos et al., 2010). Aranganathan \& Rajasree (2016) y Ramesh et al. (2020) alcanzaron un FL estable a valores de $\mathrm{pH}$ de 4.3 y 5 , respectivamente.

La materia orgánica (MO) en suspensión del FLVT fue de $171.24 \mathrm{~g} / \mathrm{l}$. Al respecto, Figueroa et al. (2015) señalan que los aminoácidos son parte esencial de la fracción activa de la MO presente en el FL orgánico. Además de la MO, los residuos fermentados de pescado contienen cantidades apreciables de nutrientes como $\mathrm{N}$, $\mathrm{P}, \mathrm{K}, \mathrm{Ca}$ y $\mathrm{Mg}$, que puedan ser aprovechadas por las plantas (Hepsibha \& Geetha, 2019). Respecto al contenido de macronutrientes, Hwang et al. (2015) señalan que las concentraciones de N, P y K de los FL comerciales tienen valores de 1000 a $2000 \mathrm{mg} / \mathrm{l}$. En la Tabla 2 se observa que el contenido de N, P y K del FLVT fue de $12040 \mathrm{mg} / 1,1189.95 \mathrm{mg} / \mathrm{l}, 5540 \mathrm{mg} / \mathrm{l}$, respectivamente. Haraldsen et al. (2011) determinaron el contenido de dos FL, uno a partir de proteína hidrolizada de residuos de salmón (HSP) con 87500 mg/l de N, 6360 mg/l de P y 14700 mg/l de K; y el otro FL, producto de una digestión anaerobia (LAD), con 2200 mg/l de N, 230 mg/l de P y 1130 mg/l de K. Casaclang et al. (2017) señalan que el contenido de $\mathrm{N}$, $\mathrm{P}$ y K de un FL a partir de hidrolizado de subproductos de tilapia fue de $2.28 \%, 1.78 \%, 0.23 \%$, respectivamente, mientras que el FL a partir de subproductos de sabalote presentó $1.95 \%$ N, $0.96 \%$ P y $0.23 \% \mathrm{~K}$.

El FLVT contiene $338.5 \mathrm{mg} / \mathrm{l}$ de Ca, $52.32 \mathrm{mg} / \mathrm{l} \mathrm{de}$ $\mathrm{Fe}, 35.5 \mathrm{mg} / \mathrm{l} \mathrm{de} \mathrm{Cu}, 68.75 \mathrm{mg} / \mathrm{l} \mathrm{de} \mathrm{Zn}, 1.95 \mathrm{mg} / \mathrm{l} \mathrm{de} \mathrm{Mn}$ y $2.59 \mathrm{mg} / \mathrm{l}$ de $\mathrm{B}$. La presencia de Ca fue menor en vísceras que en cabeza, las concentraciones de $\mathrm{Fe}, \mathrm{Cu}$ y $\mathrm{Zn}$ en vísceras fueron, mayores que en otros subproductos de pescado, debido a que estos elementos se encuentran en el hígado. De hecho, el contenido de $\mathrm{Fe}, \mathrm{Cu}, \mathrm{Mn}$ y Zn fue de 308 ppm, 7.79 ppm, 1.33 ppm y $93.67 \mathrm{ppm}$, respectivamente (Bechtel \& Oliveira, 2006; Bechtel et al., 2010). El N, P y K son móviles en el floema desde las hojas, mientras que el $\mathrm{Mg}$ lo es en menor proporción; el Ca, B, Mn y Fe son inmóviles en el floema desde las hojas. La fertilización foliar (en dosis pequeñas) se efectúa para cubrir deficiencia de micronutrientes, por lo que el tiempo para que el $50 \%$ del producto aplicado sea absorbido por la planta fue de 0.2-2 h para el N, 5-10 d para el $\mathrm{P}, 10-24 \mathrm{~h}$ para el K, 1-2 d para el Ca, 2-5 h para Mg, 1-2 d para Mn, 1-2 d para Zn y 10-20 d para el Fe (Molina, 2002).

Perfil de aminoácidos

Respecto al contenido de aminoácidos, la Tabla 3 muestra que el FLVT presentó $3.12 \mathrm{~g} / 100 \mathrm{~g}$. Inoue et al. (2013) reportaron $2.12 \mathrm{~g} / 100$ en FL de residuos de pescado. FL producidos por Kim et al. (2010) y Gwon \& Kim (2012) fueron de $5.83 \mathrm{~g} / 100 \mathrm{~g}$ y $5.93 \mathrm{~g} / 100 \mathrm{~g}$, respectivamente. Augier \& Santimone (1978) reportaron $4.1 \mathrm{~g} / 100 \mathrm{~g}$ en un fertilizante foliar a base de algas. Por otro lado, Kim et al. (2010) señalan que los FL comerciales usados en horticultura en Corea presentaron $6.81 \mathrm{~g} / 100 \mathrm{~g}$, mientras que Alarcón et al. (2010) reportan $6.39 \mathrm{~g} / 100 \mathrm{~g}$.

La presencia de peptonas, aminoácidos orgánicos y micronutrientes actúan estimulando el metabolismo y catalizando las reacciones químicas, además, los aminoácidos, junto con iones de $\mathrm{B}$ y $\mathrm{Zn}$ pueden estimular el desarrollo radicular (Frassetto, 2010). De hecho, los aminoácidos son esenciales en la fracción activa de materia orgánica e influyen en el crecimiento de las plantas dependiendo de su disponibilidad y composición. Incluso la presencia de triptófano, en pequeña cantidad, se considera como un precursor del ácido indol-3-acético, hormona de crecimiento en plantas (Augier \& Santimone, 1978; Kim et al., 2007). Metales pesados

Los vegetales asimilan $\mathrm{Hg}, \mathrm{Pb}, \mathrm{Cd}, \mathrm{Zn}$ y $\mathrm{Cu}$ y los acumulan en partes comestibles a diferentes concentraciones (Paradelo et al., 2008). Los metales pesados no afectan el crecimiento de las plantas por una disminución del potencial osmótico del sustrato, sino por su propia toxicidad. Un exceso de metales pesados o de sus quelatos solubles induce una serie de alteraciones bioquímicas y fisiológicas, entre las que se encuentra la inhibición del crecimiento de la raíz (Cartaya et al., 2008). Debido a ello, el Real Decreto 506/2013 de España señala que los productos fertilizantes elaborados con materias primas de origen animal no deben exceder los límites máximos permisibles (LMP) de metales pesados $(\mathrm{mg} / \mathrm{kg})$. El contenido de $\mathrm{Pb}, \mathrm{Cd}$ y $\mathrm{Cr}$ del FLVT (Tabla 4) estuvo por debajo de los LMP del Real Decreto Español y de los LMP (mg/l) de la normativa propuesta por la Ley Japonesa de control de fertilizantes. Kim et al. (2010) reportaron $0.26 \mathrm{mg} / \mathrm{kg}$ de $\mathrm{Cr}$ y ausencia de $\mathrm{Pb}$ y $\mathrm{Cd}$, mientras que en los FL comerciales, el contenido fue de $0.63 \mathrm{mg} / \mathrm{kg}$ de $\mathrm{Pb}, 0.08 \mathrm{mg} / \mathrm{kg}$ de $\mathrm{Cd}$ y $3.54 \mathrm{mg} / \mathrm{kg}$ de Cr.

Análisis Microbiológicos

Los resultados del análisis microbiológico se muestran en la Tabla 5. Se observa la ausencia de E. coli y Salmonella. Al respecto, Leroi (2010) 
menciona que en las operaciones de eviscerado y fileteado del pescado puede ocurrir una contaminación posterior debido a manipulaciones de operarios.

Tabla 4. Contenido de metales pesados según normativas para fertilizantes líquidos.

\begin{tabular}{|c|c|c|c|c|c|}
\hline \multirow{3}{*}{ 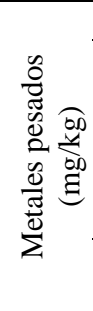 } & \multicolumn{3}{|c|}{ España } & \multirow{3}{*}{ 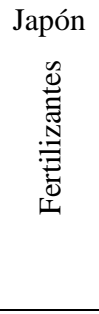 } & \multirow{3}{*}{ FLVT } \\
\hline & \multicolumn{3}{|c|}{ Fertilizante (líquido) } & & \\
\hline & $\begin{array}{c}\text { Clase } \\
\text { A }\end{array}$ & $\begin{array}{c}\text { Clase } \\
\text { B }\end{array}$ & $\begin{array}{c}\text { Clase } \\
\text { C }\end{array}$ & & \\
\hline $\mathrm{Pb}$ & 45 & 150 & 200 & 100 & 0.6 \\
\hline $\mathrm{Cd}$ & 0.7 & 2 & 3 & 5 & 0.16 \\
\hline $\mathrm{Cr}$ & 70 & 250 & 300 & 500 & 2.29 \\
\hline
\end{tabular}

FLVT: fertilizante líquido (orgánico) utilizando vísceras de trucha.

Ito (2006) señala que la presencia de E. coli y Salmonella, entre otros microorganismos patógenos, son riesgos que pueden introducirse durante la elaboración de un fertilizante orgánico mediante fermentación láctica. Por lo tanto, una limpieza y almacenamiento inadecuado puede ser fuente de contaminación de E . coli. La adición de $20 \%$ de melaza a un sustrato con y sin inóculo (tanto para un sistema anaerobio como aerobio), garantiza la ausencia de E. coli, Salmonella y orugas (Sastro et al., 2013). Al respecto, el Real Decreto 506/2013 señala que el contenido de microorganismos para fertilizantes de origen orgánico debe ser menor a $1000 \mathrm{NMP} / \mathrm{g}$ de producto elaborado para Escherichia coli, además la Salmonella debe estar ausente en $25 \mathrm{~g}$ de producto elaborado. Sin embargo, gran número de microorganismos asociados con las vísceras de pescado de agua dulce decrecen durante la fermentación exceptuando los mohos y levaduras (Rai et al., 2010). El contenido de Lactobacillus sp. en el FLVT fue de $1 \times 10^{7} \mathrm{UFC} / \mathrm{ml}$, mientras que, en el ensilado de subproductos de pescado, Berenz (1997) reporta $4.5 \times 10^{9} \mathrm{UFC} / \mathrm{g}$ al segundo día y decreció a $3.5 \times 10^{8}$ UFC/g transcurrido el tercer mes. Sastro et al. (2013) señalan que un FL elaborado vía fermentación biológica contiene $8.7 \times 10^{6} \mathrm{UFC} / \mathrm{ml}$. Al respecto Ito (2006) indica que la correlación entre el Lactobacillus sp. y el pH es importante, debido a que ocasiona la disminución del $\mathrm{pH}$ por la producción de ácido láctico y ácidos grasos de cadena corta. Respecto al contenido de mohos y levaduras, el FLVT presentó $4.5 \times 10^{3}$ UFC/ml, mientras Fernández et al. (2016) obtuvieron hasta $3.5 \times 10^{3} \mathrm{UFC} / \mathrm{g}$ en la fermentación de cabezas y vísceras de merluza (Merluccius hubbsi). Tanto las bacterias lácticas como las levaduras son responsables de la preservación, transformación y mejora en calidad organoléptica en el ensilado de subproductos de pescado (Faid et al.,1997).

\section{Fitotoxicidad}

Un bioensayo de fitotoxicidad se encarga de determinar la relación entre una dosis de la sustancia en estudio y su reacción en organismos de prueba con el fin de determinar la presencia de sustancias fitotóxicas y/o genotóxicas. La interacción de las semillas de lechuga, especie sensible a compuestos fitotóxicos, y el FLVT diluido fue positiva debido a que los compuestos de FL no interfirieron en la elongación de la radícula ni en la aparición de anormalidades durante su germinación. El índice de germinación, principal bioindicador, combina la germinación de las semillas con el crecimiento de la radícula, de esta forma, se logró obtener una estimación completa de la fitotoxicidad (Venegas et al., 2018). Además, valores elevados de CE pueden amenazar la supervivencia de los microorganismos del suelo, y reducir la calidad del FL ya que es sabido que algunos microorganismos tienen efectos positivos en la raíz de las plantas (Lakhal et al., 2017).

Las placas con alícuota de FLVT al $100 \%$ no presentaron germinación debido al $\mathrm{pH}$ de 3.9 y CE de $24.2 \mathrm{dS} / \mathrm{m}$. De igual manera, la dilución al 10\% tampoco presentó germinación debido al pH de 4.02 y $\mathrm{CE}$ de $0.8 \mathrm{dS} / \mathrm{m}$. El pH del FLVT fue alrededor de 6 cuando fue diluido en la relación fertilizante:agua de 1:1 000. Al respecto, Arnon et al. (1942) determinaron que el crecimiento óptimo de la lechuga ocurre a pH de 6. Las diluciones de $0.001 \%$ y $0.01 \%$ se encuentraron dentro del rango aceptable con valores de $\mathrm{pH}$ de $5.9 \mathrm{y}$ 5.64, respectivamente. Al-Maskri et al. (2010) categorizaron la lechuga como moderadamente tolerable a la sal y señalan que niveles de salinidad superiores a $2 \mathrm{dS} / \mathrm{m}$ y $2.6 \mathrm{dS} / \mathrm{m}$ reducen el rendimiento de la lechuga y el crecimiento de las plantas, respectivamente. Las diluciones de $10 \%$ a $0.001 \%$ presentaron un valor inferior a los límites antes señalados.

Zucconi et al. (1981) señalan que un IG mayor a $80 \%$ indica la ausencia de sustancias fitotóxicas o se encuentran a baja concentración. Por lo tanto, la dilución $0.1 \%$ con IG de $129.1 \%$ se encuentra libre de sustancias que afecten la germinación de semillas, mientras la dilución $1 \%$ presentó un IG de $61.77 \%$ e indica la presencia moderada de sustancias fitotóxicas.

Kim et al. (2010) evaluaron su FL a partir de residuos de pescado mediante una prueba de fitotoxicidad en Lepidium sativum donde, con la dilución del FL aumentada en 500 veces y 1000 veces $(0.001 \%)$, obtuvieron un IG aproximado de $60 \%$ y $80 \%$, respectivamente; mientras que dos FL comerciales para horticultura obtuvieron un IG de $60 \%$ y $80 \%$ a una dilución de $0.001 \%$. Por otra parte, Quiñonez et al. (2016) evaluaron un abono líquido vía fermentación de heces de alpaca y lactosuero bovino, la fitotoxicidad en semillas de lechuga obtuvo un IG de $94.26 \%$ a una dilución de $0.1 \%$, mientras que diluciones de $0.01 \%$ y $0.001 \%$ obtuvieron un IG menor 
a $80 \%$; y Zhu et al. (2013) obtuvieron un IG mayor a $80 \%$ diluyendo el FL a $0.01 \%$ en semillas de Lepidium sativum.

\section{Conclusiones}

La fermentación láctica permitió la estabilización y aprovechamiento del subproducto (vísceras principalmente) del procesamiento de trucha al elaborar un fertilizante orgánico líquido en un tiempo de 120 horas. El fertilizante líquido elaborado presentó similares cantidades de N. P. K, aminoácidos y microelementos a los reportados en otros fertilizantes orgánicos líquidos. Así mismo, el fertilizante orgánico líquido elaborado estuvo libre de microorganismos patógenos y el contenido de metales pesados fue menor a los límites máximos permisibles establecidos por normativas para fertilizantes. Además, las dos diluciones estudiadas estuvieron libres de sustancias fitotóxicas y no interfirieron en la germinación de semillas de lechuga. Por los resultados obtenidos, se permite proponer el uso del fertilizante líquido de vísceras de trucha en zonas agrícolas cercanas a las granjas piscícolas de trucha, lo que sugiere el uso de sistemas tecnificados de fertirrigación mediante la inyección del fertilizante líquido elaborado, en sistemas hidropónicos y acuapónicos como suplemento de micronutrientes. Adicionalmente, debido a su contenido de aminoácidos y microorganismos, también se sugiere su uso en la fertilización de estanques de tierra para incrementar la productividad primaria y como medio de cultivo alternativo para la producción de alimento vivo como microalgas (en la alimentación de las fases larvarias de peces y bivalvos como la concha de abanico). Finalmente, se recomienda el uso de este bioproceso en los subproductos del procesamiento primario de la actividad acuícola de especies amazónicas, ya que, debido al clima de la selva peruana, la fermentación puede ser llevada a cabo sin el uso de equipos costosos.

\section{Literatura citada}

Al-Maskri A., Al-Kharusi L. \& Al-Miqbali H. 2010. Effects of salinity stress on growth of lettuce (Lactuca sativa) under closed-recycle nutrient film technique. International Journal of Agriculture and Biology, 12(3): 377-380.

Alarcón-Flores M.I., Romero-González R., Frenich A.G., Vidal J.L.M. \& Reyes R.C. 2010. Rapid determination of underivatized amino acids in fertilizers by ultra high performance liquid chromatography coupled to tandem mass spectrometry. Analytical Methods, 2(11): 17451751. DOI: https://dx.doi.org/10.1039/c0ay00263a.

Aranganathan L. \& Rajasree S. R. 2016. Bioconversion of marine trash fish (MTF) to organic liquid fertilizer for effective solid waste management and its efficacy on Tomato growth. Management of Environmental Quality: An International Journal, 27(1): 93-103. DOI: https://doi.org/10.1108/MEQ-05-2015-0074.
Arnon D.I., Fratzke W.E. \& Johson C.M. 1942. Hydrogen ion concentration in relation to absorption of inorganic nutrients by higher plants. Plant Physiology, 17(4): 515524. DOI: https://doi.org/10.1104/pp.17.4.515.

Augier H. \& Santimone M. 1978. Composition en azote total, en protéines et en acides aminés protéiques de fertilisant foliaire «Goémar», à base d'algues marines. Botanica Marina, 21(6): $\quad 337-341 . \quad$ DOI: https://doi.org/10.1515/botm.1978.21.6.337.

Bechtel P.J., Morey A., Oliveira A.C.M., Wu T.H., Plante S. \& Bower C.K. 2010. Chemical and nutritional properties of pacific ocean perch (Sebastes alutus) whole fish and by-products. Journal of Food Processing and Preservation, 34: 55-72. DOI: https://doi.org/10.1111/j.1745-4549.2008.00279.x.

Bechtel P.J. \& Oliveira A.C.M. 2006. Chemical characterization of liver lipids and proteins from cold water fish species. J. Food Sci. 71: 480-485. DOI: https://doi.org/10.1111/j.1750-3841.2006.00076.x.

Berenz Z. 1997. XIII Curso Internacional Tecnología de Procesamiento de Productos Pesqueros. Callao (Perú): Instituto Tecnológico Pesquero del Perú.

Cartaya O., Marrero O., Reynaldo I. \& Peniche C. 2008. Adsorción de iones [Cu.sup.2+] por quitosana y su aplicación al suelo para la protección de las plantas ante la toxicidad del metal. Cultivos Tropicales, 29(3): 81. http://scielo.sld.cu/scielo.php?script=sci_arttext\&pid=S0 258-59362008000300015\&lng=es\&nrm=iso\&tlng=es.

Casaclang J.R., Gregorio A.C., Resuello R.B., Reyes C.J.A., Bernardo-Fernandez J. \& Gonzales-Plasus M.M. 2017. Fish hydrolysate derived from fish waste increased the growth of Kappaphycus alvarezii. AACL Bioflux, 10(5): 1150-1156.

Chapman H. \& Pratt P. 1973. Métodos de análisis para suelos, plantas y aguas. Editorial Trillas. México.

Cupe Flores B.E. \& Juscamaita Morales J.G. 2018. Tratamiento de lodos residuales de una industria cervecera a través de fermentación homoláctica para la producción acelerada de abono orgánico. Ecología aplicada, 17(1): 107-118. DOI: http://dx.doi.org/10.21704/rea.v17i1.1179.

Faid M., Zouiten A., Elmarrakchi A. \& Achkari-Begdouri A. 1997. Biotransformation of fish waste into a stable feed ingredient. Food Chemistry, 60(1): 13-18. DOI: https://doi.org/10.1016/S0308-8146(96)00291-9

Fernández-Herrero A., Fernández-Compás A., Salomone A., Vittone M. \& Manca E. 2016. Fermentación láctica del residuo de Merluccius hubbsi. Evaluación de la calidad nutricional. REDVET - Revista electrónica de Veterinaria, 17(7): 1-14.

Fernández-Salvador J., Strik B. C. \& Bryla D. R. 2015. Liquid corn and fish fertilizers are good options for fertigation in blackberry cultivars grown in an organic production system. HortScience, 50(2): 225-233. DOI: https://doi.org/10.21273/HORTSCI.50.2.225.

Figueroa J.G.S., Jung H.Y., Jeong G.T. \& Kim J.K. 2015. The high reutilization value potential of high-salinity anchovy fishmeal wastewater through microbial degradation. World J Microbiol Biotechnol, 31: 1575-1586.

Frassetto E.G. 2010. Enraizamento de estacas de Sebastiania schottiana Müll. Arg. Ciência Rural, 40(12): 2505-2509. 
Goldhor S. \& Regenstein J.M. 2007. Waste management and co-product recovery in fish processing. In: Handbook of Waste Management and Co-Product Recovery in Food Processing, Vol. 1. 389-416. Elsevier Inc.

Gwon B.G. \& Kim J.K. 2012. Feasibility study on production of liquid fertilizer in a $1 \mathrm{~m} 3$ reactor using fishmeal wastewater for commercialization. Environ. Eng. Res., 17:3-8. DOI: https://doi.org/10.4491/eer.2012.17.1.003.

Hamid A.A., Baker J. \& Bee G.H. 2002. Nutritional quality of spray dried protein hydrolysate from Black Tilapia (Oreochromis mossambicus). Food Chemistry, 78(1): 6974. DOI: https://doi.org/10.1016/S0308-8146(01)003806.

Haraldsen T.K., Andersen U., Krogstad T. \& Sørheim R. 2011. Liquid digestate from anaerobic treatment of source-separated household waste as fertilizer to barley. Waste Management \& Research, 29(12): 1271-1276. DOI: https://doi.org/10.1177/0734242X11411975.

Heinrikson R.L. \& Meredith S.C. 1984. Amino Acid Analysis by Reverse-Phase High-Performance Liquid Chromatography: Precolumn Derivatization with Phenylisothiocyanate. Analytical Biochemistry, 136(1): 65-74. DOI: https://doi.org/10.1016/00032697(84)90307-5.

Hepsibha B.T. \& Geetha A. 2019. Physicochemical characterization of traditionally fermented liquid manure from fish waste (Gunapaselam). Indian Journal of Traditional Knowledge (IJTK), 18(4): 830-836.

Holguín M.S., Caicedo L.A. \& Veloza L.C. 2009. Estabilidad de almacenamiento de ensilados biológicos a partir de residuos de pescado inoculados con bacterias ácido lácticas. Rev.Med. Vet. Zoot, 56: 95-104.

Hugli T.E. \& Moore S. 1972. Determination of the tryptophan content of proteins by ion exchange chromatography of alkaline hydrolysates. Journal of Biological Chemistry, 247(9): 2828-2834.

Hwang I.H., Aoyama H., Abe N., Matsuo T. \& Matsuto T. 2015. Subcritical hydrothermal treatment for the recovery of liquid fertilizer from scallop entrails. Environmental Technology, 36(1): 11-18. DOI: https://doi.org/10.1080/09593330.2014.934741.

ICMSF (International Commission on Microbiological Specifications for Foods). 1983. Microorganismos de los alimentos: Técnicas de análisis microbiológico. Segunda Edición. Editorial Acribia. Zaragoza.

Ito S. 2006. Caracterización y evaluación de los factores que determinan la calidad nutricional e inocuidad en la producción de fertilizantes orgánicos fermentados. M.Sc. thesis. Centro Agronómico Tropical de Investigación y Enseñanza (CATIE), Turrialba, Costa Rica.

Inoue S., Suzuki-Utsunomiya K., Komori Y., Kamijo A., Yumura I., Tanabe K., Miyawaki A. \& Koga K. 2013. Fermentation of non-sterilized fish biomass with a mixed culture of film-forming yeasts and lactobacilli and its effect on innate and adaptive immunity in mice. Journal of Bioscience and Bioengineering, 116(6): 682-687. DOI: https://doi.org/10.1016/j.jbiosc.2013.05.022.

Kim J.K., Kim J.B., Cho K.S. \& Hong Y.K. 2007. Isolation and identification of microorganisms and their aerobic biodegradation of fish-meal wastewater for liquidfertilization. Int. Biodeter. Biodegr., 59: 156-165. DOI: https://doi.org/10.1016/j.ibiod.2006.09.001.
Kim J.K., Dao V.T., Kong I.S. \& Lee H.H. 2010. Identification and characterization of microorganisms from earthworm viscera for the conversion of fish wastes into liquid fertilizer. Bioresource Technology, 101(14): 5131-5136.

DOI: https://doi.org/10.1016/j.biortech.2010.02.001

Lakhal D., Boutaleb N., Bahlaouan B., Taiek T., Fathi A., Mekouar M., Abouakil N., Lazar S., \& El Antri S. 2017. Mixture Experimental Design in the Development of a Bio Fertilizer from Fish Waste, Molasses and Scum. International Journal of Engineering Research \& Technology, 6(6), 588-594. https://www.ijert.org/mixture-experimental-design-inthe-development-of-a-bio-fertilizer-from-fish-wastemolasses-and-scum.

Leroi F. 2010. Occurrence and role of lactic acid bacteria in seafood products. Food Microbiology, 27: 698-709. DOI: https://doi.org/10.1016/j.fm.2010.05.016.

Lopes I.G., de Souza L.F., da Cruz M.C.P. \& Vidotti R.M. 2019. Composting as a strategy to recycle aquatic animal waste: Case study of a research centre in São Paulo State, Brazil. Waste Management \& Research, 37(6): 590-600. DOI: https://doi.org/10.1177/0734242X19830170.

Ministerio de la Presidencia. 2013. Real Decreto 506/2013, de 28 de junio, sobre productos fertilizantes. BOE 164, 51119-51207. España.

Molina E. 2002. Fertilización foliar de cultivos frutícolas. En: Meléndez G. \& Molina E. (Editores) Memoria, fertilización foliar: principios y aplicaciones, 85-100. Laboratorio de Suelos y Foliares. Centro de investigaciones agronómicas, Universidad de Costa Rica, San José.

Owens J.D \& Mendoza L.S. 1985. Enzymically hydrolysed and bacterially fermented fishery products. Journal of Food Technology, 20: 273-293. DOI: https://doi.org/10.1111/j.1365-2621.1985.tb00378.x.

Paradelo R., Moldes A.B., Rodríguez M. \& Barral M.T. 2008. Relación entre metales pesados y fitotoxicidad en composts. Ciencia y Tecnología Alimentaria, 6(2): 143151.

PRODUCE (MINISTERIO DE LA PRODUCCIÓN). 2018. Anuario estadístico Pesquero y Acuícola 2017. Perú.

Quiñonez-Ramirez H., Trejo-Cadillo W. \& JuscamaitaMorales. J. 2016. Evaluación de la calidad de un abono líquido producido vía fermentación homoláctica de heces de alpaca. Ecología Aplicada, 15(2): 133-142. DOI: http://dx.doi.org/10.21704/rea.v15i2.753.

Rai A.K., Swapna H.C., Bhaskar N., Halami P.M. \& Sachindra N.M. 2010. Effect of fermentation ensilaging on recovery of oil from fresh water fish viscera. Enzyme and Microbial Technology, 46(1): 9-13. DOI: https://doi.org/10.1016/j.enzmictec.2009.09.007.

Ramesh T., Amuthavalli A. \& Boopathy R. 2020. Analysis of fermented liquid fertilizer from marine crab waste. International Journal of Environment, Agriculture and Biotechnology, 5(3). DOI: https://dx.doi.org/10.22161/ijeab.53.16.

Radziemska M., Vaverková M.D., Adamcová D., Brtnický M. \& Mazur Z. 2019. Valorization of fish waste compost as a fertilizer for agricultural use. Waste and Biomass Valorization, 10(9): 2537-2545. DOI: https://doi.org/10.1007/s12649-018-0288-8. 
Sahu B.B., Sahu U., Tripathy U., Barik N.K., Agnibesh A., Paikaray A., Mohapatra S., Senapati S. \& Sundaray J.K. 2017. Fusion of sugar industry and fish processing industry waste products in developing high value organic fertilizer and feed supplement. International Journal of Fisheries and Aquatic Research, 2(4):6-18.

Sanes F.S.M., Strassburger A.S., Araújo F.B. \& Barbosa C.A. 2015. Compostagem e fermentação de resíduos de pescado para produção de fertilizantes orgánicos. Semina: Ciências Agrárias, Londrina, 36(3): 1241-1252. DOI: $\quad$ https://dx.doi.org/10.5433/16790359.2015v36n3p1241.

Sastro Y., Bakrie B. \& Sudolar N.R. 2013. The effect of fermentation method, microbes inoculation and carbon source proportion on the quality of organic fertilizer made from liquid wastes of chicken slaughterhouse. Journal of the Indonesian Tropical Animal Agriculture, 38(4): $257-$ 263. DOI: https://doi.org/10.14710/jitaa.38.4.257-263.

Sobrero M.C. \& Ronco A. 2008. Ensayo de toxicidad aguda con semillas de lechuga Lactuca sativa L. En: Castillo G. (Editor) Ensayos Toxicológicos y Métodos de Evaluación de Calidad de Aguas, 71-79. Ottawa, Canadá.

Soni S.K., Soni R. \& Janveja C. 2013. Production of fermented foods. En: Panesar P.S. \& Marwaha S.S. (Editores) Biotechnology in agriculture and food processing opportunities and challenges, 219-278. CRC Press, Boca Raton.

Spanopoulos-Hernández M., Ponce J.T., Barba G., Ruelas J.R., Tiznado M.R., Hernandez C. \& Shirai K. 2010. Producción de ensilados biológicos a partir de desechos de pescado, del ahumado de atún aleta amarilla (Thunnus albacares) y del fileteado de tilapia (Oreochromis sp), para la alimentación de especies acuícolas. Revista Mexicana de Ingeniería Química, 9(2): 167-178.

Torres C., Londoño J., Hincapié S. \& David CA. 2013. Extracción y caracterización de aceite de pescado derivado de subproductos de trucha arco iris (Oncorhynchus mykiss). Journal of Agriculture and Animal Sciences, 2(2): 34-41.
Unnisa S.A. 2015. Liquid Fertilizer from Food Waste - A Sustainable Approach. International Research Journal of Environment Sciences, 4(8): 22-25.

Valente B.S., Xavier E.G., Pereira H. da S. \& Pilotto M.V.T. 2016. Composting of marine fish residues and rice husk. Revista Brasileira de Saúde e Produção Animal, 17(2): 237-248.

Valente B.S., Andreazza R., Xavier E.G., Gomes M.C., Pereira H.D.S. \& Ávila F.D.D. 2017. Composting for valuation of marine fish waste. Revista Brasileira de Saúde e Produção Animal, 18(4): 594-603. DOI: https://doi.org/10.1590/s1519-99402017000400010.

Varnero M., Rojas C. \& Orellana R. 2007. Índices de fitotoxicidad en residuos orgánicos durante el compostaje. Revista de Ciencia del suelo y nutrición vegetal, 7(1): 28-37.

Venegas M., Leiva A.M. \& Vidal G. 2018. Influence of Anaerobic Digestion with Pretreatment on the Phytotoxicity of Sewage Sludge. Water, Air, and Soil Pollution, 229(12): 3811. DOI: https://doi.org/10.1007/s11270-018-4025-5.

Waterman J.J. 2001. Measures, stowage rate and yields of fishery products. Advisory Note No. 17, Torry Research Station, Aberdeen, Scotland, U. K.

Yamamoto M., Saleh F., Ohtsuka A. \& Hayashi K. 2005. New fermentation technique to process fish waste. Anim. Sci. J., 76: 245-248. DOI: https://doi.org/10.1111/j.17400929.2005.00262.x.

Zhu Z., Zhang F., Wang C., Ran W. \& Shen Q. 2013. Treating fermentative residues as liquid fertilizer and its efficacy onthe tomato growth. Scientia Horticulturae, 164: 492498. DOI: https://doi.org/10.1016/j.scienta.2013.10.008.

Zucconi F., Forte M., Monaco A. \& Beritodi M. 1981. Biological evaluation of compost maturity. Biocycle, 22: 27-29. 
FERTILIZANTE LÍQUIDO POR FERMENTACIÓN LÁCTICA DE SUBPRODUCTOS DE TRUCHA Agosto - Diciembre 2020

Tabla 2. Contenido de parámetros fisicoquímicos en distintos fertilizantes líquidos.

\begin{tabular}{|c|c|c|c|c|c|c|c|c|c|c|}
\hline \multirow{3}{*}{ Parámetros } & \multicolumn{10}{|c|}{ Fertilizante Líquido } \\
\hline & \multirow{2}{*}{$\begin{array}{l}\text { Sahu } \\
\text { et al. } \\
\text { (2017) }\end{array}$} & \multirow{2}{*}{$\begin{array}{l}\text { Inoue } \\
\text { et al. } \\
(2013)\end{array}$} & \multirow{2}{*}{$\begin{array}{l}\text { Sanes } \\
\text { et al. } \\
(2015)\end{array}$} & \multicolumn{2}{|c|}{$\begin{array}{l}\text { Haraldsen et al. } \\
\quad \text { (2011) }\end{array}$} & \multirow{2}{*}{$\begin{array}{c}\text { Kim } \\
\text { et al. } \\
(2010) \\
\text { FLRP }\end{array}$} & \multirow{2}{*}{$\begin{array}{l}\text { Kim et al. } \\
(2010)\end{array}$} & \multirow[t]{2}{*}{$\begin{array}{c}\text { Aranganathan } \\
\text { \& Rajasree } \\
(2016)\end{array}$} & \multirow[t]{2}{*}{$\begin{array}{l}\text { Ramesh } \\
\text { et al. } \\
(2020)\end{array}$} & \multirow[t]{2}{*}{ FLVT } \\
\hline & & & & HSP & LAD & & & & & \\
\hline $\mathrm{pH}$ & 5.03 & - & - & 6.27 & 8.02 & - & - & 4.2 & 5.0 & 4.4 \\
\hline $\mathrm{CE}(\mathrm{dS} / \mathrm{m})$ & 2.98 & - & - & - & - & - & - & 19 & 11.623 & 25.9 \\
\hline $\begin{array}{l}\text { Sólidos en } \\
\text { suspensión } \\
(\mathrm{g} / \mathrm{l})\end{array}$ & - & - & - & - & - & - & - & - & - & 199.98 \\
\hline $\begin{array}{l}\text { MO en } \\
\text { suspensión } \\
(\mathrm{g} / \mathrm{l})\end{array}$ & - & - & - & - & - & - & - & - & - & 171.24 \\
\hline $\begin{array}{l}\mathrm{N} \text { total } \\
(\mathrm{mg} / \mathrm{l})\end{array}$ & $\begin{array}{l}25 \\
600\end{array}$ & - & 15560 & 87500 & 2200 & 15700 & 38000 & 10000 & 2100 & 12040 \\
\hline $\begin{array}{l}\text { P total } \\
(\mathrm{mg} / \mathrm{l})\end{array}$ & 9600 & 1022 & 2450 & 6360 & 230 & 3100 & 28300 & 3900 & 4100 & 1189.95 \\
\hline $\begin{array}{l}\mathrm{K} \text { total } \\
(\mathrm{mg} / \mathrm{l})\end{array}$ & $\begin{array}{l}12 \\
000\end{array}$ & 1530 & 1240 & 14700 & 1130 & 4500 & 30400 & 3700 & 2700 & 5540 \\
\hline $\begin{array}{l}\text { Ca total } \\
(\mathrm{mg} / \mathrm{l})\end{array}$ & $\begin{array}{l}15 \\
500\end{array}$ & 373 & 5030 & 310 & 730 & - & - & 1400 & $5830^{*}$ & 338.5 \\
\hline $\begin{array}{l}\text { Mg total } \\
(\mathrm{mg} / \mathrm{l})\end{array}$ & 700 & 1260 & 520 & 520 & 140 & - & - & 5200 & $1283^{*}$ & 615 \\
\hline $\begin{array}{l}\mathrm{Na} \text { total } \\
(\mathrm{mg} / \mathrm{l})\end{array}$ & - & 1930 & 720 & 9600 & 640 & - & - & - & $1500^{*}$ & 4500 \\
\hline $\mathrm{Fe}(\mathrm{mg} / \mathrm{l})$ & - & 20.4 & - & - & - & - & - & $50.51^{*}$ & $57.30^{*}$ & 52.35 \\
\hline $\mathrm{Cu}(\mathrm{mg} / \mathrm{l})$ & - & 0.41 & - & 0.12 & 0.19 & - & $2.24^{*}$ & $0.61^{*}$ & $2.31^{*}$ & 35.5 \\
\hline $\mathrm{Zn}(\mathrm{mg} / \mathrm{l})$ & - & 5.25 & - & 1.58 & 1.15 & $1.72^{*}$ & $3.51^{*}$ & $5.66^{*}$ & $9.64^{*}$ & 68.75 \\
\hline $\mathrm{Mn}(\mathrm{mg} / \mathrm{l})$ & - & 4.11 & - & $<0.01$ & - & - & - & $2.81^{*}$ & $17.33^{*}$ & 1.95 \\
\hline $\mathrm{B}(\mathrm{mg} / \mathrm{l})$ & - & 1 & - & 0.65 & 0.47 & - & - & - & - & 2.59 \\
\hline
\end{tabular}

Tabla 3. Contenido de aminoácidos en distintos fertilizantes orgánicos.

\begin{tabular}{|c|c|c|c|c|c|c|c|}
\hline \multirow[b]{3}{*}{$\begin{array}{l}\text { Aminoácido } \\
(\mathrm{g} / 100 \mathrm{~g})\end{array}$} & \multirow[b]{3}{*}{$\begin{array}{l}\text { Inoue } \\
\text { et al. } \\
(2013)\end{array}$} & \multirow[b]{3}{*}{$\begin{array}{c}\text { Augier \& } \\
\text { Santimone } \\
\text { (1978) }\end{array}$} & \multicolumn{3}{|c|}{ Fertilizante líquido } & \multirow[b]{3}{*}{$\begin{array}{l}\text { Hepsibha } \\
\text { \& Geetha } \\
\text { et al } \\
\text { (2019) }\end{array}$} & \multirow{3}{*}{ FLVT } \\
\hline & & & \multirow[b]{2}{*}{$\begin{array}{l}\text { Kim } \\
\text { et al. } \\
(2010)\end{array}$} & \multicolumn{2}{|c|}{ Comercial } & & \\
\hline & & & & $\begin{array}{l}\text { Kim } \\
\text { et al. } \\
\text { (2010) }\end{array}$ & $\begin{array}{l}\text { Alarcón } \\
\text { et al. } \\
\text { (2010) }\end{array}$ & & \\
\hline Ác. Aspártico & 0.235 & 0.31 & 0.49 & 0.58 & 1.32 & 0.124 & 0.28 \\
\hline Treonina & 0.101 & 0.18 & 0.21 & 0.21 & 0.1 & 0.0346 & 0.16 \\
\hline Serina & 0.085 & 0.18 & 0.21 & 0.23 & - & 0.0023 & 0.15 \\
\hline Ac. glutámico & 0.297 & 1.29 & 0.78 & 0.89 & 0.02 & 0.247 & 0.21 \\
\hline Prolina & 0.147 & 0.19 & 0.5 & 0.61 & 0.51 & 0.298 & - \\
\hline Glicina & 0.255 & 0.25 & 1.06 & 1.25 & 0.04 & 0.203 & 0.02 \\
\hline Alanina & 0.204 & 0.31 & 0.7 & 0.98 & 0.83 & - & 0.39 \\
\hline Valina & 0.112 & 0.19 & 0.17 & 0.24 & 0.91 & 0.356 & 0.19 \\
\hline Isoleucina & 0.077 & 0.14 & 0.15 & 0.13 & 0.03 & 0.705 & 0.12 \\
\hline Leucina & 0.152 & 0.28 & 0.27 & 0.26 & 0.04 & 0.394 & 0.26 \\
\hline Tirosina & 0.011 & 0.1 & 0.07 & 0.05 & 0.01 & 0.306 & 0.19 \\
\hline Fenilalanina & 0.073 & 0.19 & 0.18 & 0.18 & 0.02 & 0.291 & 0.13 \\
\hline Histidina & 0.096 & 0.07 & 0.2 & 0.25 & 0.03 & 0.129 & 0.06 \\
\hline Lisina & 0.158 & 0.15 & 0.39 & 0.53 & 1.13 & 0.703 & 0.21 \\
\hline Arginina & 0.034 & 0.22 & 0.31 & 0.35 & 0.05 & 0.129 & 0.63 \\
\hline Cisteína & 0.018 & 0.04 & 0.06 & 0.04 & - & - & - \\
\hline Metionina & 0.059 & 0.05 & 0.06 & 0.01 & 1.35 & 0.297 & 0.06 \\
\hline Triptófano & 0.007 & 0.016 & 0.02 & 0.02 & - & 0.123 & 0.06 \\
\hline Total & 2.12 & 4.15 & 5.83 & 6.81 & 6.39 & 4.34 & 3.12 \\
\hline
\end{tabular}


Tabla 5. Características microbiológicas de distintos fertilizantes líquidos.

\begin{tabular}{|c|c|c|c|c|}
\hline \multirow{3}{*}{ Contenido } & \multicolumn{4}{|c|}{ Fuente de fertilizante líquido } \\
\hline & \multicolumn{2}{|c|}{ Sastro et al. (2013) } & \multirow{2}{*}{$\begin{array}{c}\text { Hepsibha } \\
\text { \& Geetha } \\
\text { (2019) } \\
\text { Residuos } \\
\text { de } \\
\text { pescado }\end{array}$} & \multirow{2}{*}{$\begin{array}{c}\text { FLVT } \\
\text { Vísceras } \\
\text { de trucha }\end{array}$} \\
\hline & Comercial & $\begin{array}{c}\text { Residuos } \\
\text { de pollo }\end{array}$ & & \\
\hline $\mathrm{pH}$ & 3.9 & 4.5 & 4.15 & 4.4 \\
\hline Lactobacillus sp. (UFC/ml) & $8.7 \times 10^{6}$ & $2.0 \times 10^{8}$ & $1.38 \times 10^{4}$ & $1 \times 10^{7}$ \\
\hline Mohos y levaduras (UFC/ml) & $8.5 \times 10^{6}$ & $3.5 \times 10^{2}$ & $1.73 \times 10^{2}$ & $4.5 \times 10^{3}$ \\
\hline Escherichia coli $(\mathrm{NMP} / \mathrm{ml})$ & Ausencia & Ausencia & Ausencia & Ausencia \\
\hline Salmonella sp. en $25 \mathrm{ml}$ & Ausencia & Ausencia & Ausencia & Ausencia \\
\hline
\end{tabular}

FLVT: fertilizante líquido (orgánico) utilizando vísceras de trucha.

\footnotetext{
${ }^{1}$ Laboratorio de Biotecnología Ambiental y Biorremediación. Facultad de Ciencias, Universidad Nacional Agraria La Molina. Av La Molina s/n, Lima 12, Perú. 20080699@lamolina.edu.pe.

${ }^{2}$ Departamento Académico de Acuicultura e Industrias Pesqueras. Facultad de Pesquería, Universidad Nacional Agraria La Molina. Av La Molina s/n, Lima 12, Perú. droldan@lamolina.edu.pe.

${ }^{3}$ Departamento Académico de Biología, Facultad de Ciencias. Universidad Nacional Agraria La Molina. Av La Molina s/n, Lima 12, Perú. jjm@lamolina.edu.pe.
} 